\title{
Auditory fatigue among call center operators with headset
}

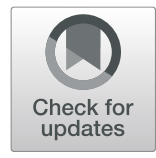

\author{
Pretty Omar Afifi ${ }^{1}$, Tayseer Taha Tayseer Abdel Rahman ${ }^{1 *}$ (D) and Ahmed Gamal Khafagy ${ }^{2}$
}

\begin{abstract}
Background: To assess whether call center operators are subject to or suffer from any auditory fatigue when compared to normal subjects. A prospective case-control study where twenty-eight call center operators (10 females and 18 males) with an age range from 25 to 46 years and twenty controls ( 12 females and 8 males) with an age range from 23 to 44 years were included. All call center operators use a headset for up to $8 \mathrm{~h}$ daily for a minimum of two consecutive years. Audiological assessments were done to all participants that included pure tone audiometry (PTA), speech audiometry, tympanometry, and acoustic reflexes. Moreover, transient-evoked otoacoustic emission (TEOAE) absolute threshold and distortion product otoacoustic emission (DPOAE) signal to noise thresholds as well as contralateral suppression of TEOAEs and DPOAEs were recorded for both groups.

Results: There was no significant variation in auditory performances detected with either PTA or OAE (TEOAEs and DPOAEs) test. Besides, there was no statistically significant difference in OAEs (TEOAEs and DPOAEs) with contralateral suppression for both the call center operators and control groups. However, call center operators expressed a feeling of tiredness.

Conclusions: There was no detectable central or peripheral auditory fatigue experienced by the call center operators when using headsets. However, their sensation of auditory fatigue could be due to cognitive fatigue rather than noise-induced fatigue.
\end{abstract}

Keywords: Auditory fatigue, Call center, Otoacoustic emission, Efferent pathway

\section{Background}

Noise-induced hearing loss could be defined as damage to hearing because of sustained high levels of noise that have a direct toxic effect on the hair cell mechanism of the cochlea. This could lead to irreversible cochlear dysfunction whereas auditory fatigue is a more subtle concept. In the beginning, there is temporary hearing loss (auditory fatigue) which is located at the level of the peripheral receptors. In a study done by Liberman and Dodds [1], they discovered shortening of the rootlets of the stereocilia with more flexibility to the structures and swellings underneath the inner hair cells cause a massive release of glutamate within the synaptic cleft. This is

\footnotetext{
*Correspondence: tayseerhesham2005@gmail.com

'Otorhinolaryngology Department, Ain Shams University, Faculty of medicine, Cairo, Egypt

Full list of author information is available at the end of the article
}

mainly observed after moderate-noise exposures, which lead to the establishment of a peripheral auditory fatigue.

Cochlear trauma might happen easily through headsets and might lead to acoustic shocks [2, 3]. This could be seen in $12 \%$ of the equivalent diffuse field that exceeded $80 \mathrm{dBA}$ and maximum levels of up to $90 \mathrm{dBA}$ [4]. Call center operators usually work with the headset in an open space, which means that they would be exposed to environmental noise as well as noise received through the headsets.

The current internationally recognized reference screening tool to assess noise-induced hearing loss (NIHL) is subjective pure-tone audiometry (PTA) thresholds. The peripheral auditory receptors send a piece of information to the central auditory system, which at the same time analyzes and could correct this information for subtle ear dysfunctions or metabolic fatigue to ensure the highest

\section{Springer Open}

(๑) The Author(s). 2020 Open Access This article is licensed under a Creative Commons Attribution 4.0 International License, which permits use, sharing, adaptation, distribution and reproduction in any medium or format, as long as you give appropriate credit to the original author(s) and the source, provide a link to the Creative Commons licence, and indicate if changes were made. The images or other third party material in this article are included in the article's Creative Commons licence, unless indicated otherwise in a credit line to the material. If material is not included in the article's Creative Commons licence and your intended use is not permitted by statutory regulation or exceeds the permitted use, you will need to obtain permission directly from the copyright holder. To view a copy of this licence, visit http://creativecommons.org/licenses/by/4.0/. 
possible level of performance [5, 6]. Subsequently, PTA results may underestimate NIHL due to these retro-cochlear compensatory mechanisms.

Contralateral suppression of transient-evoked otoacoustic emissions (TEOAEs) and distortion product otoacoustic emissions (DPOAEs) were hypothesized as an objective test for measuring auditory fatigue. These tests are more objective and sensitive than PTA due to eliciting the trigger of the efferent reflex (ER), so the data obtained from these tests is not affected by retro-cochlear compensatory mechanisms [7].

The efferent auditory system contains many neural pathways, which transfer the auditory information from the lower brainstem to the cochlea [8]. The medial olivocochlear neurons consist of large and myelinated fibers, which originate around the medial nuclei of the superior olivary complex and finally terminate on outer hair cells $(\mathrm{OHCs})$ of the organ of Corti $[8,9]$. Most of $\mathrm{OHCs}$ have binaural input; however, some project ipsilaterally and contralaterally [10]. During acoustic stimulation, there is an inhibitory response from the medial olivocochlear system (MOCS) which is recorded by the decrease in the level of OAEs in normal-hearing individuals $[11,12]$. That is to say, the MOC pathway is the main efferent pathway and it is crossed to end in the contralateral OHCs. Moreover, by subtracting the level of otoacoustic emission in the presence of the suppressor stimulus from the level of otoacoustic emission in the absence of suppressor stimulus could be measured.

Contralateral suppression of OAEs in different groups of the population has been reported as a very important tool in many studies $[13,14]$. It mainly measures the amount of protection that exists in individuals when the efferent system is activated (suppression of emissions). Moreover, contralateral suppression of OAEs is noninvasive, less time-consuming, and a precise measure. This makes it, by many researchers, the preferred tool to evaluate the auditory efferent system. Despite its wide application, there is a scarcity of literature to explore the efferent system function in call center workers using TEOAEs and DPOAEs.

Therefore, this study was done to assess efferent system function in call center operators using TEOAEs and DPOAEs, as well as assessing the role of olivocochlear bundle (OCB) in these individuals. The main goal of this study was to determine whether call center operators present subjectively and/or objectively evidence of peripheral and/or central auditory fatigue.

\section{Methods}

\section{Study sample}

This study consisted of 28 operators ( 10 females and 18 males) with an age range from 25 to 46 years as a study group and 20 volunteers (12 females and 8 males) with an age range from 23 to 44 years serving as a control group. All call center operators (study group) use a headset for up to $8 \mathrm{~h}$ daily for 6 days a week for a minimum of two consecutive years (exposure duration from 2 to 4 years). This study was conducted in the audiovestibular unit from the period January 2019-February 2020. A written consent was taken from all subjects for participation in this study.

Full medical history was gathered for all participants including any previous noise exposure or acute acoustic trauma. Any participant with a possible ear infection, impacted wax, abnormalities in the external auditory canal, ear disease, previous ear operations, neurologic disease, vertigo, and/or abnormalities in the tympanometry (type B or C) were excluded from this study. All of these might affect the hearing performance of these individuals.

Audiological assessments were done on all participants that include pure-tone, speech audiometry, tympanometry, and acoustic reflexes. All 48 participants (28 call center workers and 20 volunteers) were also tested with otoacoustic emissions including contralateral suppression of transient-evoked otoacoustic emissions (TEOAEs) and distortion product otoacoustic emissions (DPOAEs). OAEs were conducted for patients whose audiological findings indicate normal middle ear function. Patients with abnormal middle ear functions shown by otological examination or tympanometry were excluded from this study.

\section{Procedure \\ Audiological evaluations}

To evaluate hearing sensitivity, pure tone thresholds were obtained at octave intervals between 250 and 8000 $\mathrm{Hz}$ for air conduction while between $500 \mathrm{~Hz}$ and $4 \mathrm{kHz}$ for bone conduction thresholds using a calibrated twochannel clinical audiometer GSI 61 clinical audiometer (Grason-Stadler-A Division of VIASYS HealthcareDenmark). The audiometer was calibrated according to the procedure described in EN 60645-1/AINSI S3.6 type 4. The ascending descending method with $5 \mathrm{~dB}$ step was used. Normal audiometric thresholds were accepted at different frequencies ( $\leq 25 \mathrm{~dB} \mathrm{HL})$.

Tympanometry measurements to assess middle ear status using a middle ear analyzer GSI TympStar Version 2 (Grason-Stadler, Madison, WI) with a probe tone frequency of $226 \mathrm{~Hz}$ were performed. Ipsilateral and contralateral acoustic reflexes thresholds were measured for 500 , 1000, 2000, and $4000 \mathrm{~Hz}$.

\section{Otoacoustic emissions (TEOAEs and DPOAEs)}

Otoacoustic emissions TEOAEs (absolute thresholds) and DPOAEs (signal to noise thresholds), as well as contralateral suppression of TEOAEs and DPOAEs, were recorded with the biologic-evoked potential systemNavigator PRO (model 580, NAVPR2, version 7.0.0). A 
standard TEOAE/DPOAE probe tip was inserted in the individual's ear canal.

For TEOAE recording, an acoustic stimulus was delivered to the ear with an average intensity of $85 \mathrm{~dB}$ SPL. Emission was recorded at five frequency bands over a frequency range of 1.0 to $4.0 \mathrm{kHz}$. DPOAEs (DP-grams) were measured in an audiometric cabin using the NAVP R2 Scout (Version 4.0) at 1000, 1501, 2002, 3174, 4004, and $6384 \mathrm{~Hz}$ (f2). The L1 was set $=65 \mathrm{~dB} \mathrm{SPL}$ and the $\mathrm{L} 2=55 \mathrm{~dB}$ SPL, the $\mathrm{f} 1 / \mathrm{f} 2$ ratio $=1.22$, and the noise floor was set to $\leq-5 \mathrm{~dB}$ SPL. Recorded measures were echo levels for TEOAE and signal-to-noise levels for DPOAE. A response was considered positive at a given frequency band, if it measured $3 \mathrm{~dB}$ or above (pass response) [15]. The Grason-Stadler Incorporated-61 diagnostic audiometer at $60 \mathrm{~dB}$ HL generated the contralateral broad-band noise for OAE suppression. It is presented contralaterally where this level will not provoke acoustic reflex.

Otoacoustic emissions (TEOAEs and DPOAEs) data obtained without/with contralateral stimulation were compared for both study and control groups. TEOAE/ DPOAE measurements were performed just after the PTA and tympanometry measurements on the same day.

\section{Test environment}

An acoustically sound-treated room was used for testing with ambient noise levels within permissible limits as per ANSI S3.1 (1991). Two-room set-up was used for PTA while a single-room set-up for both tympanometry and OAEs tests.

\section{Statistical analysis}

All data were stored and analyzed in a Statistical Package for the Social Sciences (SPSS) file version 19.0 (Inc.; Chicago, IL, USA). Descriptive statistics including means and standard deviations were used for both call center workers and volunteers. A standard $t$ test was used for comparison between the control and study groups. The student's $t$ test was used to compare between TEOAE/ DPOAE data obtained without contralateral stimulation with data obtained with contralateral stimulation. All tests were two-tailed $t$ test, and $p$ values $<0.05$ were considered significant.

\section{Results}

This study consisted of 28 operators (10 females and 18 males) with a mean age of $32 \pm 5.27$ and 20 volunteers (12 females and 8 males) with a mean age of $27.5 \pm 5.33$ serving as a control group.

The epidemiologic classification showed non-significant differences in distribution between study and control groups. Table 1 shows the demographic data in both study and control groups.
Table 1 Epidemiologic data in both study and control groups

\begin{tabular}{llll}
\hline Variable & $\begin{array}{l}\text { Study group } \\
(\boldsymbol{n}=\mathbf{2 8})\end{array}$ & $\begin{array}{l}\text { Control group } \\
(\boldsymbol{n}=\mathbf{2 0})\end{array}$ & $\boldsymbol{p}$ value \\
\hline Age (years) & $32 \pm 5.27$ & $27.5 \pm 5.33$ & 0.94 \\
Sex male/female & $18(64 \%) / 10(36 \%)$ & $8(40 \%) / 12(60 \%)$ & 0.82 \\
\hline
\end{tabular}

\section{Pure-tone audiometry}

All the participants had normal peripheral hearing in both ears, which was measured by pure-tone thresholds, less than $20 \mathrm{~dB} \mathrm{HL}$ in both ears (at frequency range $0.25-8 \mathrm{kHz}$ ), as well as lack of any middle ear pathology on the day of testing which was measured by immittancemetry (bilateral normal middle ear functions).

There was a similarity in hearing levels between control and study groups at all of the tested frequencies (Fig. 1 and Table 2). However, there was a visible difference at 4 and $8 \mathrm{kHz}$, but this difference did not reach significance $(p=0.11)$. This mostly could be explained by older age of the study group if compared to the control group (mean age of study was 32 vs. 27.5 for controls).

\section{Otoacoustic emissions (TEOAEs and DPOAEs)}

Otoacoustic emissions TEOAEs (absolute thresholds) and DPOAEs (signal to noise thresholds), as well as contralateral suppression of TEOAEs and DPOAEs, were recorded for both study and control groups. As shown in Table 3, otoacoustic emissions TEOAEs (absolute thresholds) and DPOAEs (signal to noise thresholds) without suppression showed no statistical difference between groups, whatever the frequency tested. TEOAEs in the study group had lower echo levels at mainly frequency bands 2000, 3000, and $4000 \mathrm{kHz}$ compared to the control group; however, these differences did not reach significant levels. As regards DPOAEs, its levels were also reduced in the study group at all frequencies except at $1003 \mathrm{~Hz}$.

Table 4 showed otoacoustic emissions TEOAEs (absolute thresholds) and DPOAEs (signal to noise thresholds) with suppression of otoacoustic emissions (TEOAEs and DPOAEs) for both groups. The differences were not statistically significant at any of the frequencies tested between both groups. In the study group, they had a higher suppression when compared to the control group at most frequency bands either in TEOAEs or DPOAEs. However, these differences did not reach significant levels.

\section{Discussion}

The plasticity of the central auditory system could mask some degree of cochlear defects due to peripheral auditory fatigue, which could make them undetectable by PTA. To investigate whether this phenomenon, OAE (TEOAEs and DPOAEs) tests were for assessing peripheral cochlear dysfunction. The neuronal circuit triggered 


\section{PTA threshold Study and Control Groups}

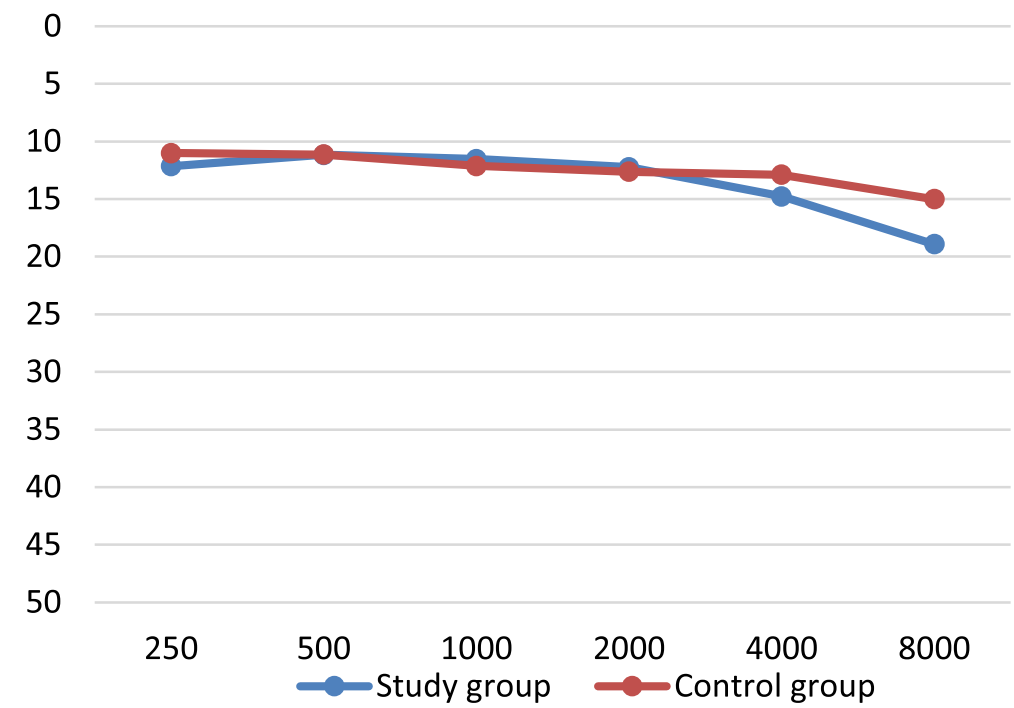

Fig. 1 Mean pure tone thresholds (dB HL) for both study and control groups

by the OAEs consists of the physiological function of inner hair cells, afferent fibers, the olivocochlear complex, the efferent fibers, and the middle-ear muscles reflex threshold $[5,6,16]$.

Peripheral and central auditory fatigue based on two complementary hearing tests (PTA and OAEs) were examined in this study. There was no significant difference found in PTA results between both call center operators and the control group. Moreover, there was no obvious auditory fatigue using PTA in call center operators (study group) which in term indicated preserved hearing performance (Fig. 1, Table 2).

This study was done to assess the efferent system function in call center operators in comparison to normal volunteers using TEOAEs and DPOAEs and the role of the olivocochlear bundle (OCB) in both groups using contralateral suppression of TEOAEs and DPOAEs.

Table 2 Mean pure tone thresholds ( $\mathrm{dB} \mathrm{HL}$ ) for both study and control groups

\begin{tabular}{lllll}
\hline Freq. (Hz) & $\begin{array}{l}\text { Study group } \\
\text { Mean + SD } \\
\text { dB HL }\end{array}$ & $\begin{array}{l}\text { Control group } \\
\text { Mean + SD } \\
\text { dB HL }\end{array}$ & $\boldsymbol{t}$ value & $\boldsymbol{p}$ \\
\hline 250 & $12.14 \pm 6.31$ & $11.00 \pm 5.91$ & 0.898 & 0.372 \\
500 & $11.16 \pm 4.37$ & $11.13 \pm 4.16$ & 0.040 & 0.968 \\
1000 & $11.52 \pm 3.92$ & $12.13 \pm 3.90$ & 0.749 & 0.456 \\
2000 & $12.23 \pm 4.94$ & $12.63 \pm 5.06$ & 0.380 & 0.705 \\
4000 & $14.77 \pm 6.67$ & $12.88 \pm 5.76$ & 0.082 & 0.935 \\
8000 & $18.91 \pm 3.57$ & $15.00 \pm 6.30$ & 0.067 & 0.947 \\
\hline
\end{tabular}

There was no statistically significant difference between call center operators in comparison to normal volunteers at most of the frequencies tested in both TEOAEs and DPOAEs. However, there was a slightly higher suppression of TEOAEs and DPOAEs in call center operators. For TEOAE, results showed that although there was higher suppression (better MOC system) for call center operators when compared to normal volunteers, it did not reach statistical significance at all frequencies. In

Table 3 Comparison between TEOAEs (absolute thresholds) and DPOAEs (signal to noise threshold levels) in study and control groups

\begin{tabular}{ccccc}
\hline Freq. (Hz) & $\begin{array}{l}\text { Study group } \\
\text { Mean } \pm \text { SD }\end{array}$ & $\begin{array}{l}\text { Control group } \\
\text { Mean } \pm \text { SD }\end{array}$ & t-value & $\mathbf{P}$ \\
\hline TEOAEs & & & & \\
1000 & $11.05 \pm 3.86$ & $12.09 \pm 3.04$ & 1.309 & 0.194 \\
1500 & $10.03 \pm 4.56$ & $10.42 \pm 3.13$ & 1.916 & 0.058 \\
2000 & $10.02 \pm 3.94$ & $12.96 \pm 2.35$ & 1.336 & 0.185 \\
3000 & $9.85 \pm 3.70$ & $11.57 \pm 1.82$ & 1.139 & 0.258 \\
4000 & $10.04 \pm 2.98$ & $12.23 \pm 2.80$ & 1.332 & 0.186 \\
DPOAEs & & & & \\
1003 & $17.52 \pm 5.52$ & $14.09 \pm 5.89$ & 0.358 & 0.721 \\
1409 & $18.60 \pm 4.95$ & $19.56 \pm 4.83$ & 0.950 & 0.345 \\
2000 & $17.87 \pm 5.74$ & $18.55 \pm 5.55$ & 0.581 & 0.563 \\
2822 & $17.75 \pm 4.16$ & $19.80 \pm 4.57$ & 0.052 & 0.959 \\
3991 & $17.91 \pm 6.49$ & $20.17 \pm 5.06$ & 0.604 & 0.548 \\
5649 & $18.84 \pm 6.51$ & $20.07 \pm 5.30$ & -1.419 & 0.159 \\
\hline
\end{tabular}


Table 4 Comparison between TEOAEs (absolute thresholds) and DPOAEs (signal to noise threshold levels) with contralateral suppression in study and control groups

\begin{tabular}{cllll}
\hline Freq. (Hz) & $\begin{array}{l}\text { Study group } \\
\text { Mean } \pm \text { SD }\end{array}$ & $\begin{array}{l}\text { Control group } \\
\text { Mean } \pm \text { SD }\end{array}$ & $\boldsymbol{t}$ value & $\boldsymbol{p}$ \\
\hline TEOAEs & & & & \\
1000 & $10.41 \pm 3.83$ & $9.68 \pm 3.07$ & 0.988 & 0.326 \\
1500 & $10.48 \pm 4.44$ & $11.01 \pm 3.10$ & 1.855 & 0.067 \\
2000 & $9.65 \pm 3.66$ & $10.57 \pm 2.34$ & 1.399 & 0.165 \\
3000 & $9.50 \pm 3.73$ & $10.18 \pm 1.87$ & 1.064 & 0.290 \\
4000 & $9.45 \pm 3.09$ & $8.276 \pm 2.77$ & 1.129 & 0.262 \\
DPOAEs & & & & \\
1003 & $16.73+5.48$ & $14.71+5.86$ & 0.023 & 0.982 \\
1409 & $17.59+5.05$ & $18.91+5.40$ & -1.223 & 0.225 \\
2000 & $17.08+5.71$ & $18.20+5.53$ & -0.961 & 0.339 \\
2822 & $16.9+4.15$ & $17.40+4.63$ & -0.455 & 0.650 \\
3991 & $16.74+6.30$ & $16.77+5.09$ & -0.029 & 0.977 \\
5649 & $17.81+6.05$ & $16.68+5.23$ & -2.23 & 0.976 \\
\hline
\end{tabular}

addition, the same results were observed for DPOAE measure (Fig. 2, Tables 3 and 4). These findings reflected that these subjects could have stronger medial efferent feedback on the auditory periphery.

Similarly, Atchariyasathian et al. [16] had investigated the hearing performance of thirty-two workers who were exposed to noise using both PTA and DPOAEs and concluded that DPOAE is superior in the evaluation of auditory fatigue in those persons. He also concluded that both study and control groups do not show any significant difference based on the PTA results; however, there was only a decrease in DPOAE amplitudes seen at $4000 \mathrm{~Hz}$ and
$6000 \mathrm{~Hz}$. Another study performed by Kumar et al. [17] on rock musicians showed higher suppression effect across frequencies in musicians when compared to nonmusicians (better protection to the auditory system across different frequencies). He concluded that rock musicians are having a better efferent system compared to nonmusicians.

Sound conditioning is playing an important role in strengthening the central auditory pathways and this could be the probable reason for the higher suppression effect found in the study group. In a study done by Eldredge and Miller [18, 19] in various animal models, they found that sound conditioning has ameliorated the damaging effect of noise trauma. MOC plays an important protective functional role and this was shown in by "toughening" of OHCs themselves [20]. Furthermore, there was another finding of the present study, in which there was more suppression effect in call center operators. This could be explained that call center operators were usually exposed to a constant dose of low-level noise, which in turn lead to sound conditioning of their ears and thus increased ability to suppress otoacoustic emissions. This reflected probably that these individuals had better protection for the auditory system across different frequencies (better efferent system).

All these components are considered to make up the peripheral receptor except for the nuclei of the olivocochlear complex. The OAEs results are not influenced by upper central stages (dorsal cochlear nucleus and inferior colliculus) due to the neuronal structure of the ER. Therefore, to detect peripheral auditory fatigue with high sensitivity, OAE measurements are used [7]. However, only small and insignificant variations in ER thresholds were measured in this study.

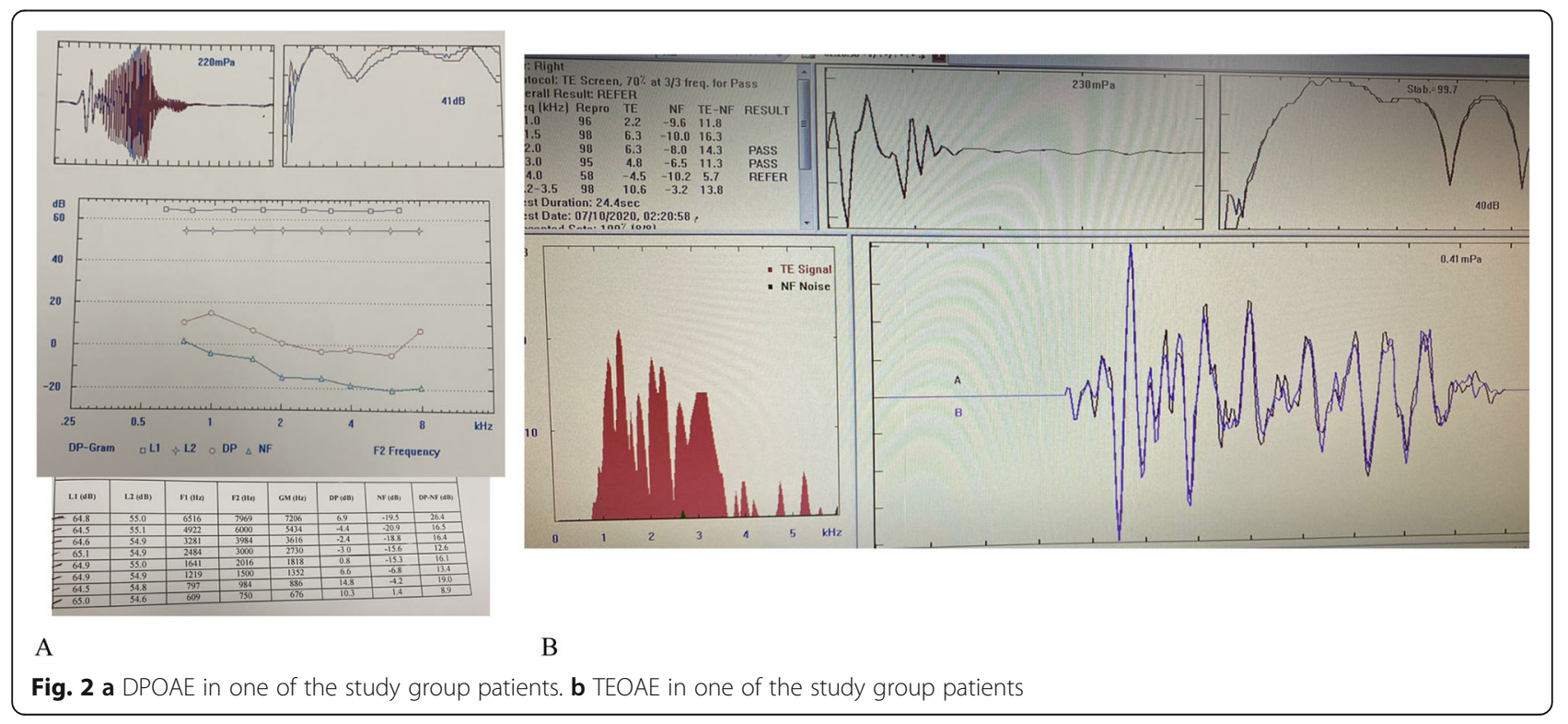


In our study and based on its results, all call center operators have not experienced either central or peripheral auditory fatigue. This could lead to the conclusion that noise equivalent exposure received through the headset (the mean $8 \mathrm{~h}$ ) was considerably lower than the noise levels commonly recorded in factories.

Despite these findings, the sensation of general fatigue and as well as auditory fatigue (in some operators) was reported in call center operators. This could be explained that it might be related to cognitive fatigue or emotional exhaustion, which is usually caused by heavy mental workload, and to call center's work-specific stressors [21, 22]. Therefore, using other tools may be needed to investigate the emotional dimension of the job in these call center operators and how it influences perceived fatigue.

There was no detectable central or peripheral auditory fatigue experienced by the call center operators when using headsets. However, their sensation of auditory fatigue could be due to cognitive fatigue rather than noise-induced fatigue.

\section{Abbreviations}

TEOAE: Transient-evoked otoacoustic emissions: DPOAE: Distortion product otoacoustic emissions; PTA: Pure tone audiometry; ER: Efferent reflex; dB $H L$ : Decibel normal hearing level

\section{Acknowledgements}

None

\section{Authors' contributions}

P.A. and A. K.: the idea of research, collecting cases, and testing. T. A.: analysis of the results and data processing, revision, and writing. The authors read and approved the final manuscript.

\section{Funding}

None

\section{Availability of data and materials}

Available.

\section{Ethics approval and consent to participate}

Ethical committee of Maghrabi Hospital-Qatar: No: ENT-5-2018Q. Consent obtained from the study participants before research; informed written consent is available.

\section{Consent for publication}

Not applicable as no data of patients were published.

\section{Competing interests}

No conflict of interest.

\section{Author details}

${ }^{1}$ Otorhinolaryngology Department, Ain Shams University, Faculty of medicine, Cairo, Egypt. ${ }^{2}$ Otorhinolaryngology Department, Ain Shams University, Cairo, Egypt.

Received: 25 April 2020 Accepted: 29 September 2020

Published online: 27 October 2020

\section{References}

1. Liberman MC, Dodds LW (1987) Acute ultrastructural changes in acoustic trauma: serial- section reconstruction of stereocilia and cuticular plates. Hear Res. 26(1):45-64

2. Westcott M (2006) Acoustic shock injury (ASI). Acta Otolaryngol. 126:54-58
3. Beyan A, Demiral Y, Cimrin A, Ergor A (2016) Call centers and noise-induced hearing loss. Noise Health. 18(81):113-116

4. Trompette N, Chatillon J (2012) Survey of noise exposure and background noise in call centers using headphones. J Occup Environ Hyg. 9(6):381-386

5. Finlayson PG, Kaltenbach JA (2009) Alterations in the spontaneous discharge patterns of single units in the dorsal cochlear nucleus following intense sound exposure. Hear Res. 256(1-2):104-117

6. Syka J (2002) Plastic changes in the central auditory system after hearing loss, restoration of function, and during learning. Physiol Rev. 82(3):601-636

7. Venet T, Campo P, Rumeau C, Thomas A, Parietti-Winkler C (2014) One-day measurement to assess the auditory risks encountered by noise-exposed workers. Int J Audiol. 53(10):737-744

8. Warr WB, Guinan JJ Jr (1979) Efferent innervation of the organ of corti: two separate systems. Brain Res. 173:152-155

9. Warr WB, Guinan JJ, White JS (1986) Organization of the efferent fibres: the lateral and medial olivocochlear systems. In: Hoffman DW, Bobbin RP (eds) Altschuler R.A. The Cochlea. Raven Press; New York, Neurobiology of Hearing, pp 333-348

10. Liberman MC (1988) Response properties of cochlear efferent neurons: monaural vs. binaural stimulation and the effects of noise. J Neurophysiol. 60:1779-1798

11. Collet L, Kemp DT, Veuillet E, Duclaux R, Moulin A, Morgon A (1990) Effects of contralateral auditory stimuli on active cochlear micro-mechanical properties in human subjects. Hear Res. 43:251-261

12. Hood L, Berlin Cl, Hurley A, Cecola RP, Bell B (1996) Contralateral suppression of transient-evoked otoacoustic emissions in humans: intensity effects. Hear Res. 101:113-118

13. Garinis AC, Glattke T, Cone-Wesson BK (2008) TEOAE suppression in adults with learning disabilities. Int J Audiol. 47:607-614

14. Geven LI, Wit HP, de Kleine E, van Dijk P (2012) Wavelet analysis demonstrates no abnormality in contralateral suppression of otoacoustic emissions in tinnitus patients. Hear Res. 286:30-40

15. Maxon AA, White K, Vohr B, Behrans T (1993) Using transient evoked otoacoustic emission for neonatal hearing screening. Br. J. Audiol 27: 149-153

16. Atchariyasathian V, Chayarpham S, Saekhow S (2008) Evaluation of noiseinduced hearing loss with audiometer and distortion product otoacoustic emissions. J Med Assoc Thail. 91:1066-1071

17. Kumar P, Grover V, Publius A S, Sanju HK, Sinha S (2016) Assessment of rock musician's efferent system functioning using contralateral suppression of otoacoustic emissions. World Journal of Otorhinolaryngology - Head and Neck Surgery

18. Eldredge DH, Covell WP, Gannon RP (1959) Acoustic trauma following intermittent exposure to tones. Ann Otol Rhinol Laryngol. 68:723-732

19. Miller JD, Watson CS, Covell WP (1963) Deafening effects of noise on the cat. Acta Otolaryngol Suppl. 176:1-91

20. Kujawa SG, Liberman MC (1999) Long-term sound conditioning enhances cochlear sensitivity. J Neurophysiol. 82:863-873

21. Mellor D, Moore KA, Siong ZMB (2015) The role of general and specific stressors in the health and well-being of call centre operators. Work. 52: $31-43$

22. Lewig KA, Dollard MF (2003) Emotional dissonance, emotional exhaustion and job satisfaction in call centre workers. Eur J Work Organ Psychol. 12(4): 366-392

\section{Publisher's Note}

Springer Nature remains neutral with regard to jurisdictional claims in published maps and institutional affiliations. 